\title{
Limb Salvage Resection of Massive Dedifferentiated Thigh Liposarcoma in a Patient Lost to Follow-Up
}

Nicholas J. Calvo ${ }^{1}$, Adam J. Mann ${ }^{1}$, Miguel Lopez-Viego 1, 2 , Thomas Genuit ${ }^{1}$

1. Surgery, Florida Atlantic University Charles E. Schmidt College of Medicine, Boca Raton, USA 2. Surgery, Bethesda Hospital East, Baptist Health South Florida, Boynton Beach, USA

Corresponding author: Nicholas J. Calvo, ncalvo2017@health.fau.edu

\begin{abstract}
Dedifferentiated liposarcoma (DDL) is a rare soft tissue tumor that represents a non-lipogenic progression of well-differentiated liposarcoma (WDL). Unlike WDL, DDL has the propensity for metastasis and is associated with an increased incidence of local recurrence. For DDL of the extremities that is resectable with acceptable functional outcomes, treatment includes primary surgical resection with negative margins. Although rare due to advances in reconstructive techniques, amputation for DDL of the extremities should be considered in which appropriate tumor resection cannot be performed without adequate preservation of limb function. We present the clinical progression of a patient with a large DDL of the right thigh who was initially lost to follow-up, but ultimately underwent delayed definitive therapy with the intent for limb salvage. This case illustrates the importance of assessing neurovascular, osseous, and soft tissue involvement to properly predict and preserve limb function while achieving adequate tumor resection.
\end{abstract}

Categories: General Surgery, Oncology

Keywords: dedifferentiated liposarcoma, extremity liposarcoma, dedifferentiated extremity liposarcoma, limbsalvage surgery, soft tissue sarcoma, well-differentiated liposarcoma

\section{Introduction}

Liposarcomas are rare soft tissue tumors that account for a worldwide incidence of 0.7 cases per 100,000 persons per year, but they are among the most common types of soft tissue sarcomas (15\%) [1]. Welldifferentiated liposarcoma (WDL) and dedifferentiated liposarcoma (DDL) comprise 50-70\% of liposarcomas. DDL represents a non-lipogenic progression of WDL that is often found in association with precursor WDL tumors. DDL is associated with a worse prognosis and decreased survival due to its propensity for metastasis and increased incidence of local recurrence [2,3]. Management of localized liposarcomas includes radical surgical resection with clear margins, as well as adjuvant or neoadjuvant radiation. Rates of local and/or distant therapy failure and prognosis are primarily related to age at diagnosis, tumor location and size, stage at initial presentation, histologic grade, heterologous differentiation, completeness of initial resection, need

Received 10/05/2020 Review began 10/08/2020 Review ended 11/29/2020 Published 02/15/2021

\section{() Copyright 2021}

Calvo et al. This is an open access article distributed under the terms of the Creative Commons Attribution License CC-BY 4.0., which permits unrestricted use, distribution, and reproduction in any medium, provided the original author and source are credited. for contiguous organ resection, and primary anatomical site [4-7]. Notably, poorer outcomes are associated with retroperitoneal tumors [6].

We present a patient with a large DDL of the right thigh. The patient was initially lost to follow-up, but eventually underwent delayed definitive therapy with the intent of limb salvage. This case illustrates the clinical progression of a DDL of the thigh and the importance of assessing neurovascular, osseous, and soft tissue involvement to properly predict and preserve limb function while achieving adequate tumor resection.

\section{Case Presentation}

A 70-year-old female patient with a past medical history of hypertension, hyperlipidemia, diabetes mellitus, and obesity presented to the emergency department with a painful anterior right thigh mass measuring approximately $25 \mathrm{~cm}$ in length. At an outside facility 2.5 years prior, magnetic resonance imaging (MRI) without contrast of the right thigh identified a heterogeneous mass with a lipogenic and a non-lipogenic component measuring $20 \times 6 \times 5 \mathrm{~cm}$ (Figures 1,2). According to the medical record, the mass had been present for years without significant changes and was causing no symptoms. An incisional biopsy of the mass, at that time, revealed adipose tissue with fibrous septations and atypical cells with enlarged hyperchromatic nuclei. Fluorescence in situ hybridization (FISH) showed MDM2 gene locus amplification, but tests for CDK4 and p16 were not performed. The biopsy and imaging findings were suggestive of DDL, rather than other lipomatous tumors (i.e., WDL, lipoma, dysplastic lipoma, spindle cell lipoma, or inflammatory myofibroblastic tumor). However, the patient was discharged with instructions to follow-up with surgery and medical oncology but was lost to follow-up. 


\section{Cureus}

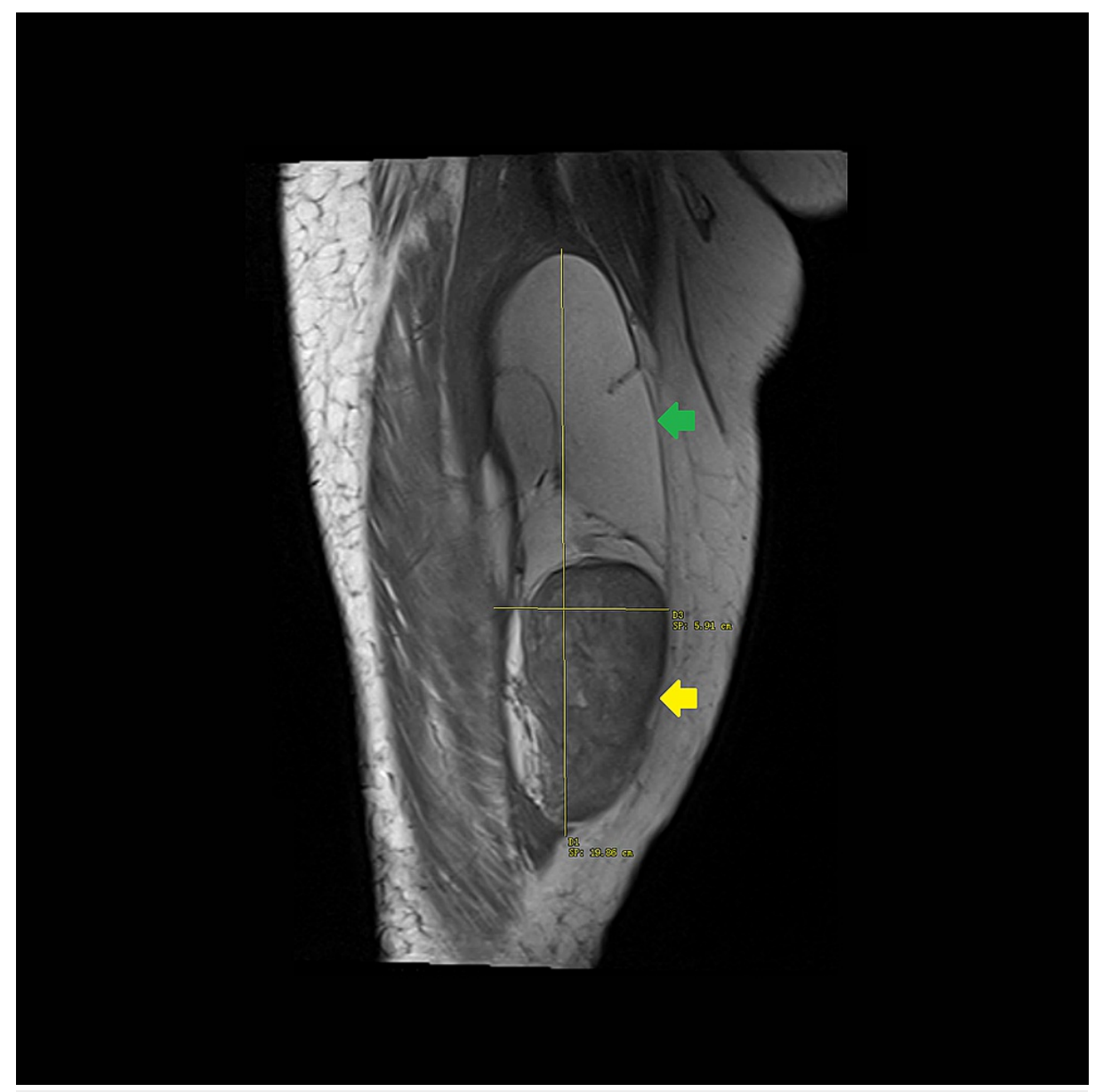

FIGURE 1: T1-weighted turbo spin echo MRI without contrast of the right thigh in coronal plane. A $20 \times 6 \times 5 \mathrm{~cm}$ multi-loculated mass with a superior lipogenic component (green arrow) and inferior non-lipogenic component measuring $9 \times 5 \times 5 \mathrm{~cm}$ (yellow arrow).

MRI, magnetic resonance imaging 


\section{Cureus}

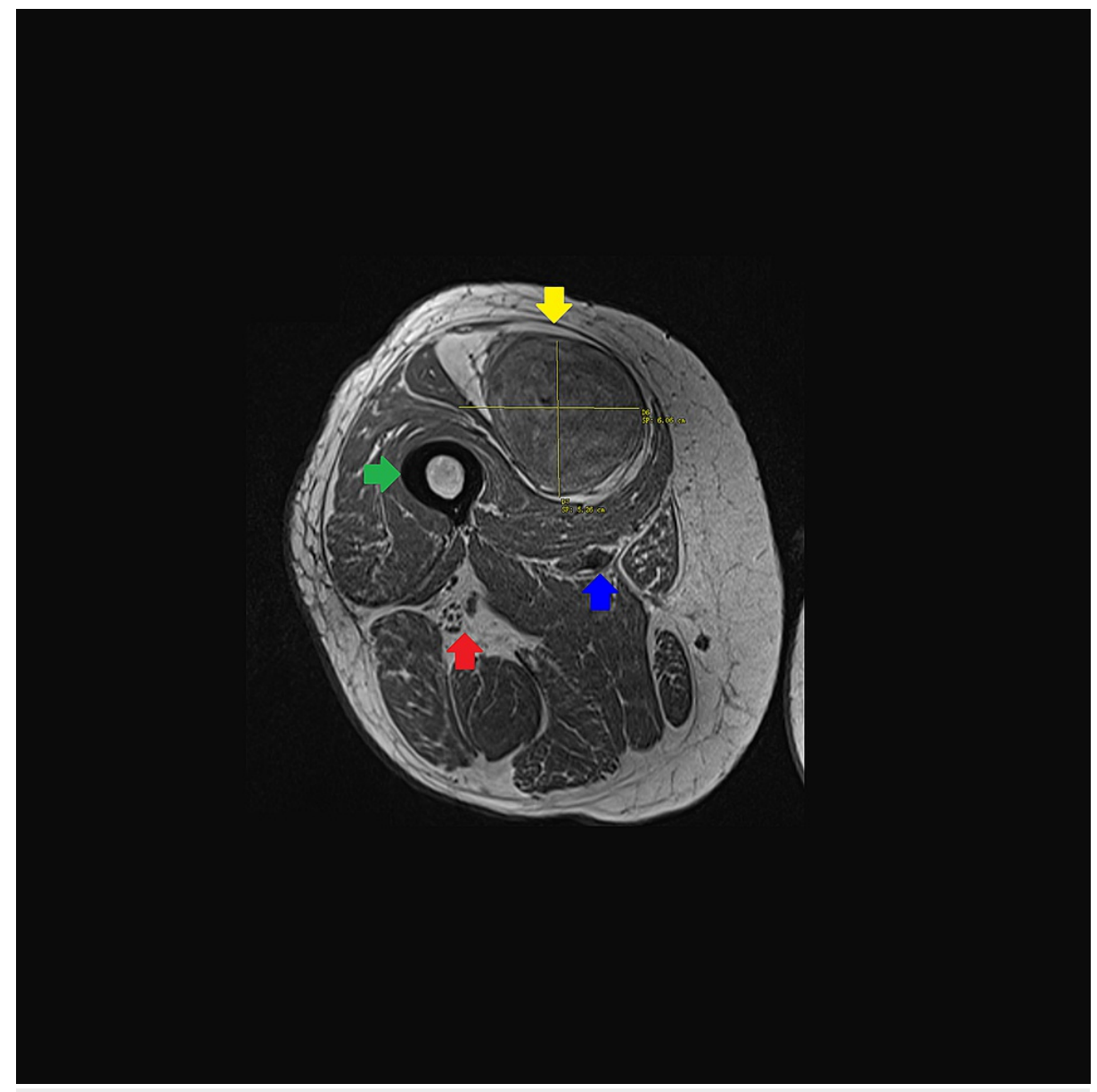

FIGURE 2: T1-weighted turbo spin echo MRI without contrast of the right thigh in axial plane. A $20 \times 6 \times 5 \mathrm{~cm}$ multi-loculated mass (yellow arrow) with a superior lipogenic component and inferior non-lipogenic component $(9 \times 5 \times 5 \mathrm{~cm})$. There is no invasion of the femur (green arrow), profunda femoris artery/vein (red arrow), or superficial femoral artery/vein and posterior division of the femoral nerve (blue arrow). The tumor displaces the rectus femoris medially and the vastus intermedius posteriorly.

MRI, magnetic resonance imaging

Subsequently, the patient presented to an outside facility six months prior to the current hospitalization with enlargement of the thigh mass, moderate pain, tingling, and decreased motor strength in the right anterior thigh compartment. An MRI with gadolinium contrast of the right thigh revealed enlargement of the mass to $23 \times 12.5 \times 8.5 \mathrm{~cm}$ with a centrally enhancing non-lipogenic component and peripheral lipogenic components (Figures 3,4 ). In accordance with the guidelines for management of liposarcomas, she was referred to a tertiary care center with a multidisciplinary sarcoma team for management, but she was again lost to follow-up. 


\section{Cureus}

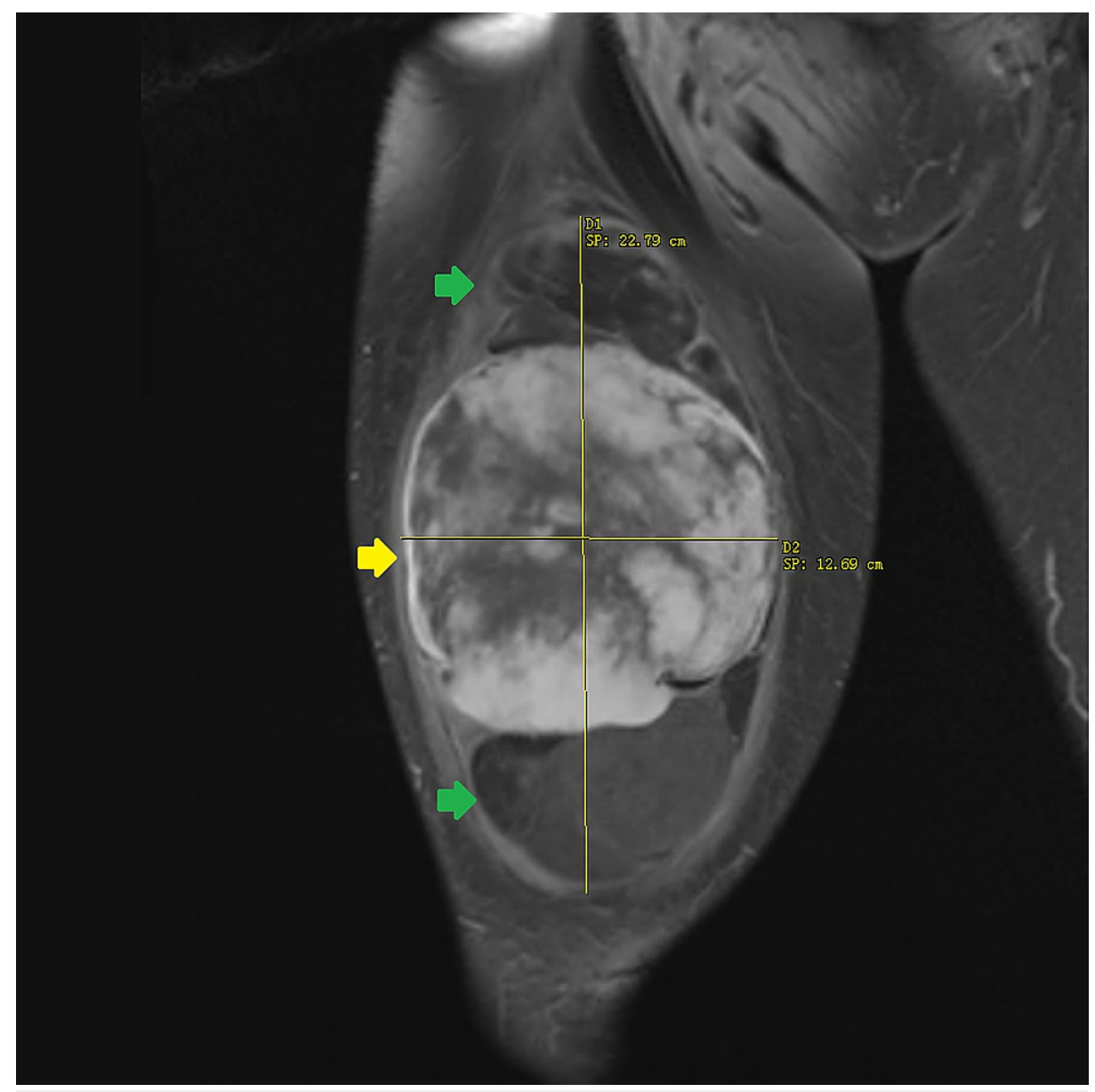

FIGURE 3: T1-weighted turbo spin echo MRI with gadolinium contrast of the right thigh in coronal plane. A $23 \times 12.5 \times 8.5 \mathrm{~cm}$ heterogeneous mass with a peripheral fat component (green arrows) and central nonlipogenic enhancing component measuring $12.6 \times 12.6 \times 8.3 \mathrm{~cm}$ (yellow arrow).

MRI, magnetic resonance imaging 


\section{Cureus}

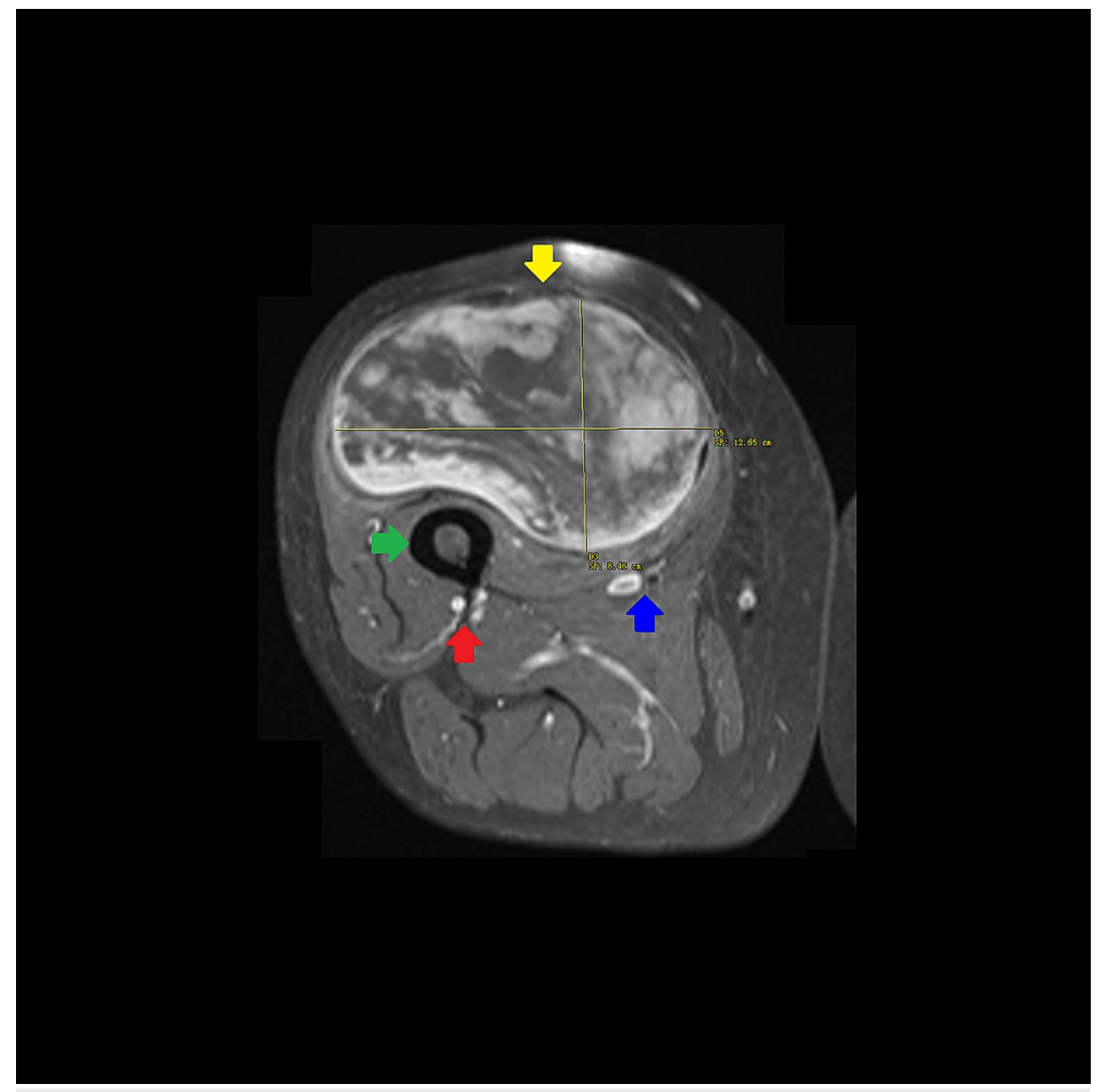

FIGURE 4: T1-weighted turbo spin echo MRI with gadolinium contrast of the right thigh in axial plane. A $23 \times 12.5 \times 8.5 \mathrm{~cm}$ heterogeneous mass (yellow arrow) abutting but not infiltrating the femur (green arrow). There is a peripheral fat component and central non-lipogenic enhancing component measuring $12.6 \times 12.6 \times 8.3 \mathrm{~cm}$. There is no invasion of the profunda femoris artery/vein (red arrow), or superficial femoral artery/vein and posterior division of the femoral nerve (blue arrow). The rectus femoris muscle is partially encased by the tumor. The sartorius and vastus muscles are partially encased and posteriorly displaced by the tumor.

MRI, magnetic resonance imaging

During the current hospitalization, the patient presented to the emergency room with deteriorating right thigh function, severe pain, paresthesia, and diminished muscle strength. The patient denied fever, weight loss, and/or any other significant symptoms. The patient was in distress due to the pain in her right thigh. The anterior right thigh mass measured $25 \mathrm{~cm}$ in length and was firm and tender to palpation. Range of motion was limited due to pain in the right lower extremity at the hip to approximately 30-105 degrees and at the knee to approximately 75-105 degrees. Muscle strength was also diminished in the right lower extremity with respect to hip flexion, hip extension, knee flexion, and knee extension. The patient had full range of motion and muscle strength at the left lower extremity and the remaining joints of the right lower extremity, as well as intact sensation and pulses in both lower and upper extremities. Inguinal lymph nodes were soft, mobile, and $<1 \mathrm{~cm}$ in diameter. The remainder of the examination was unremarkable. MRI with gadolinium contrast showed enlargement of the right thigh mass to $26 \times 19 \times 14 \mathrm{~cm}$ (previously measuring $23 \times 12.5 \times 8.5 \mathrm{~cm}$ six months ago) with inhomogeneous nodular enhancement and peripheral fat densities (Figures 5, 6). There was no involvement of the femur, profunda femoris artery/vein, posterior division of the femoral nerve, and/or superficial femoral artery/vein (Figure 6). The rectus femoris muscle was completely encased by the tumor. The sartorius, vastus medialis, and vastus intermedius muscles were nearly completely encased by the tumor. The vastus lateralis was partially encased and posteriorly displaced by the 


\section{Cureus}

tumor. There was no tumor involvement beyond the anterior thigh compartment. There was no inguinal or pelvic lymphadenopathy. Computed tomography (CT) of chest, abdomen, and pelvis with contrast showed no metastases or other acute processes.

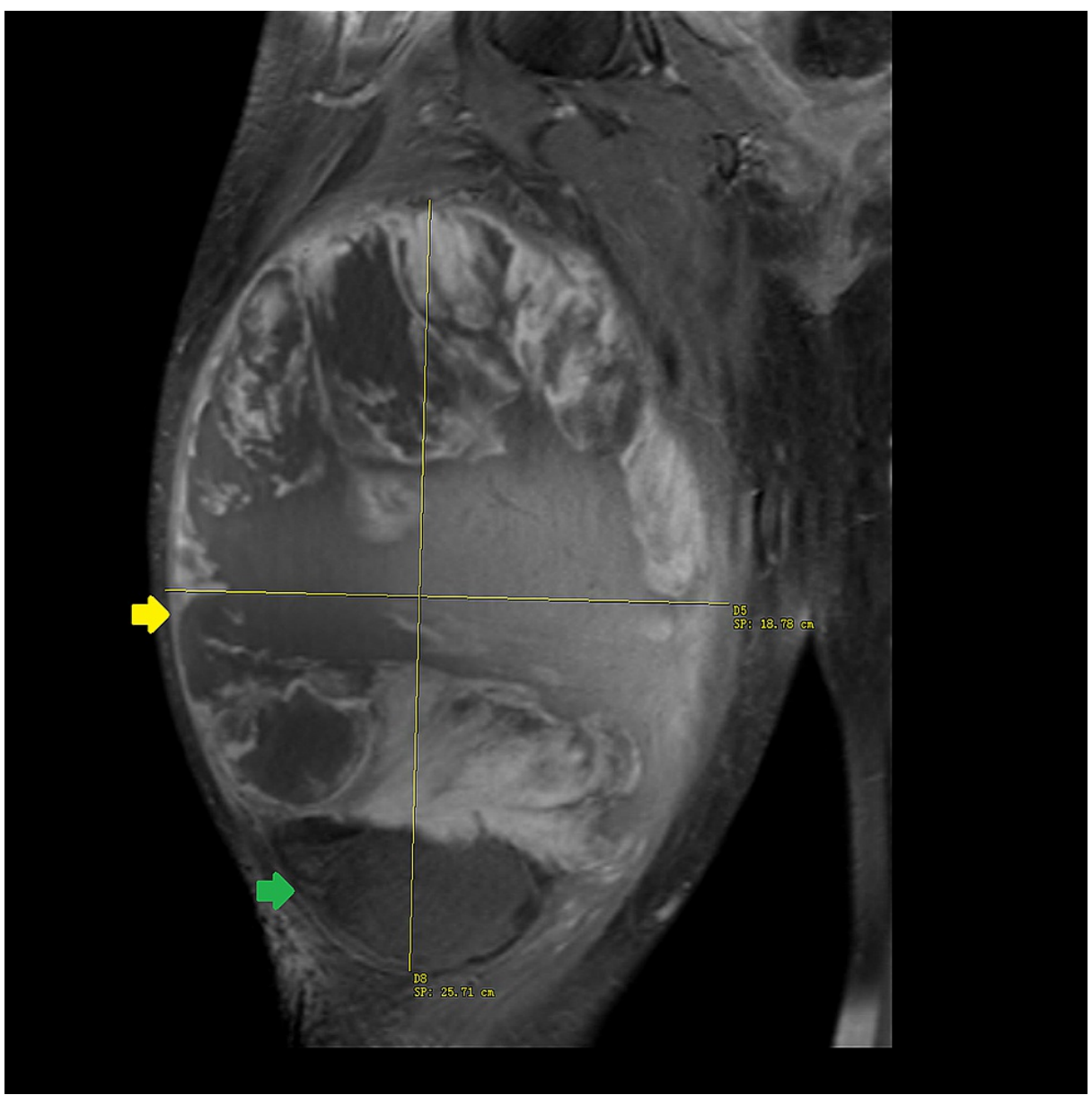

FIGURE 5: T1-weighted turbo spin echo MRI with gadolinium contrast of the right thigh in coronal plane. An anterior compartment mass measuring $26 \times 19 \times 14 \mathrm{~cm}$. The central non-lipogenic component measures $24 \times 19 \times 14 \mathrm{~cm}$ (yellow arrow) and demonstrates irregular, inhomogeneous nodular peripheral enhancement. Peripheral fat densities are present (green arrow).

MRI, magnetic resonance imaging 


\section{Cureus}

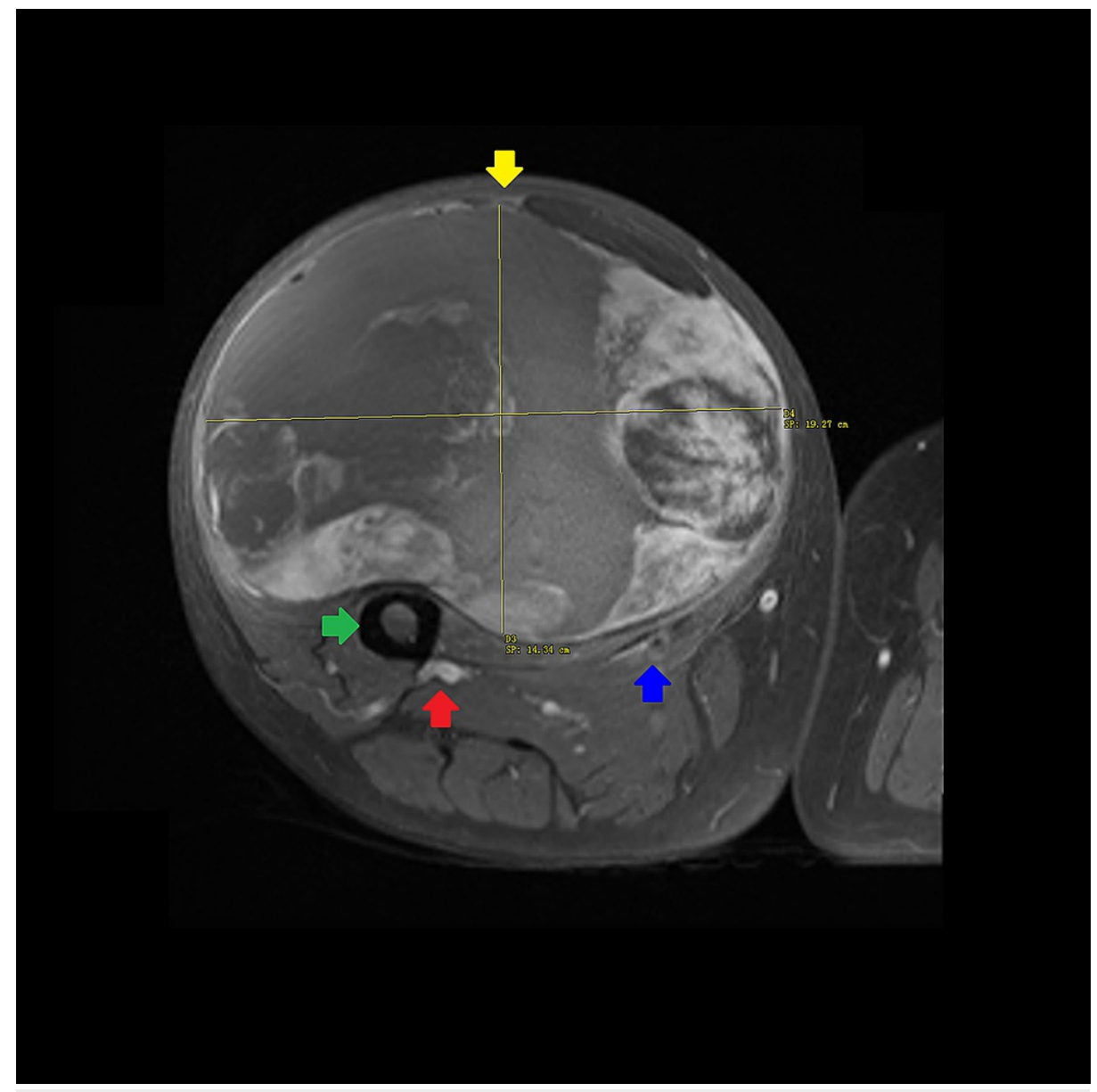

FIGURE 6: T1-weighted turbo spin echo MRI with gadolinium contrast of the right thigh in axial plane. An anterior compartment mass (yellow arrow) measuring $26 \times 19 \times 14 \mathrm{~cm}$. The central non-lipogenic component measures $24 \times 19 \times 14 \mathrm{~cm}$ and demonstrates irregular, inhomogeneous nodular peripheral enhancement. Peripheral fat densities are present. There is no invasion of the femur (green arrow), profunda femoris artery/vein (red arrow), or superficial femoral artery/vein and posterior division of the femoral nerve (blue arrow). The rectus femoris muscle is completely encased by the tumor. The sartorius, vastus medialis, and vastus intermedius muscles are almost completely encased by the tumor. The vastus lateralis is partially encased and posteriorly displaced by the tumor. There is no tumor involvement beyond the anterior thigh compartment.

\section{Treatment}

Despite the tumor's significant size, it was determined that limb salvage should still be possible as there was no neurovascular encasement or metastatic disease and tumor resection could likely be achieved with adequate preservation of right lower extremity function. Given the patient's history of medical noncompliance with follow-up, the decision was made to proceed with surgical intervention during this admission rather than referral to another facility with a multidisciplinary sarcoma team [8].

After sedation and intubation, the patient was placed in a supine position and her right lower extremity was draped and prepped above the ankle. An elliptical incision was made around the palpable border of the mass and the well-circumscribed, lobulated, soft, rubbery mass was dissected with a margin of surrounding soft tissue/muscle. Intraoperative evaluation revealed that the tumor completely invaded the rectus femoris and almost completely invaded the sartorius, vastus medialis, and vastus intermedius muscles. This portion of the tumor was resected en bloc with a margin of the surrounding muscle which did allow for the preservation 


\section{Cureus}

of some musculature. An unidentified branch of the femoral nerve (most likely the branch to the rectus femoris) was completely encased within the tumor and was resected en bloc (Figures 7, 8). Medially the tumor was adjacent to the superficial femoral artery but without encasement or direct invasion, allowing its preservation. The tumor did not extend past the anterior thigh compartment. Intraoperative frozen section of the deep margins showed no malignancy.

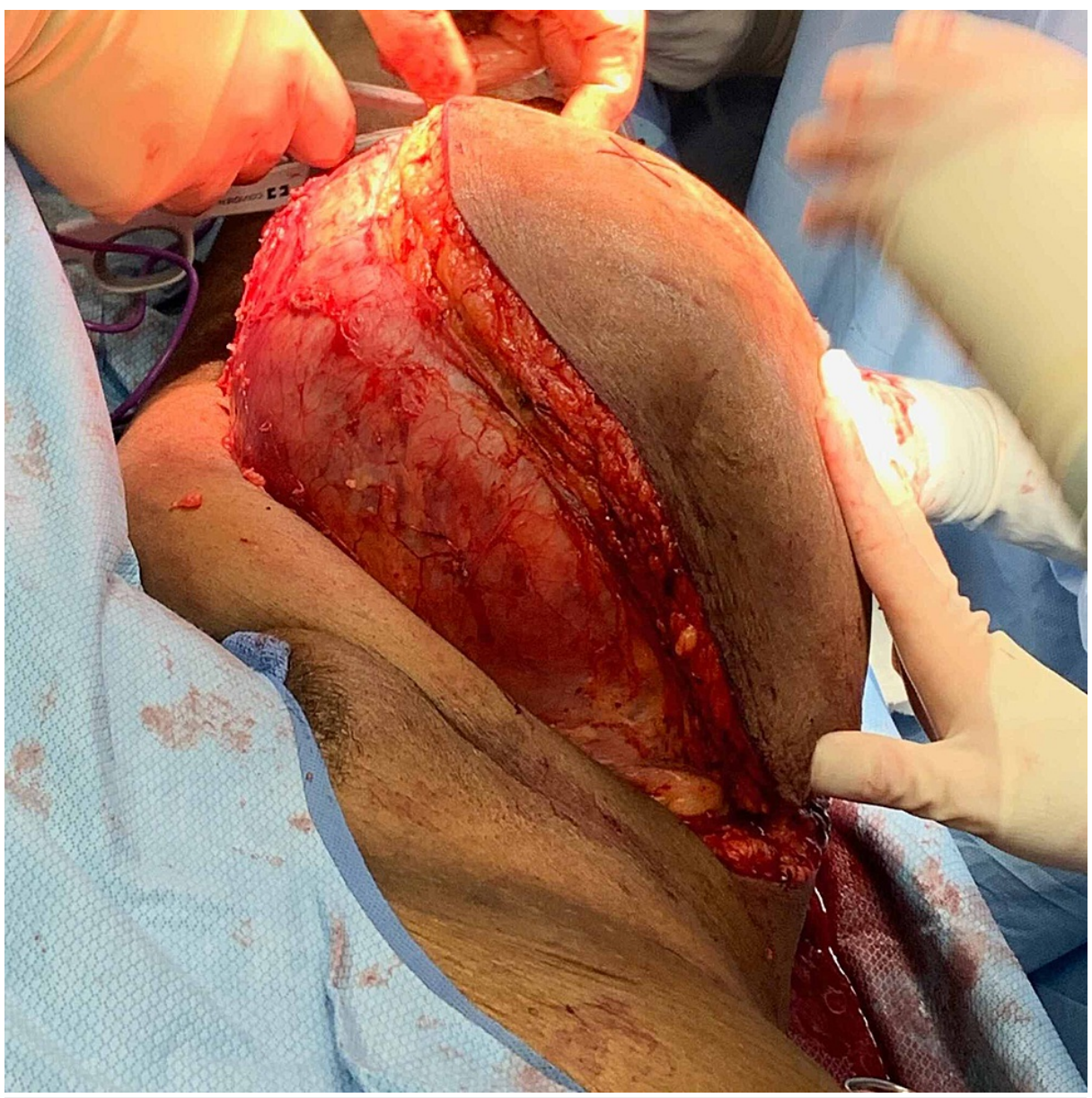

FIGURE 7: Tumor resection of the anterior right thigh mass.

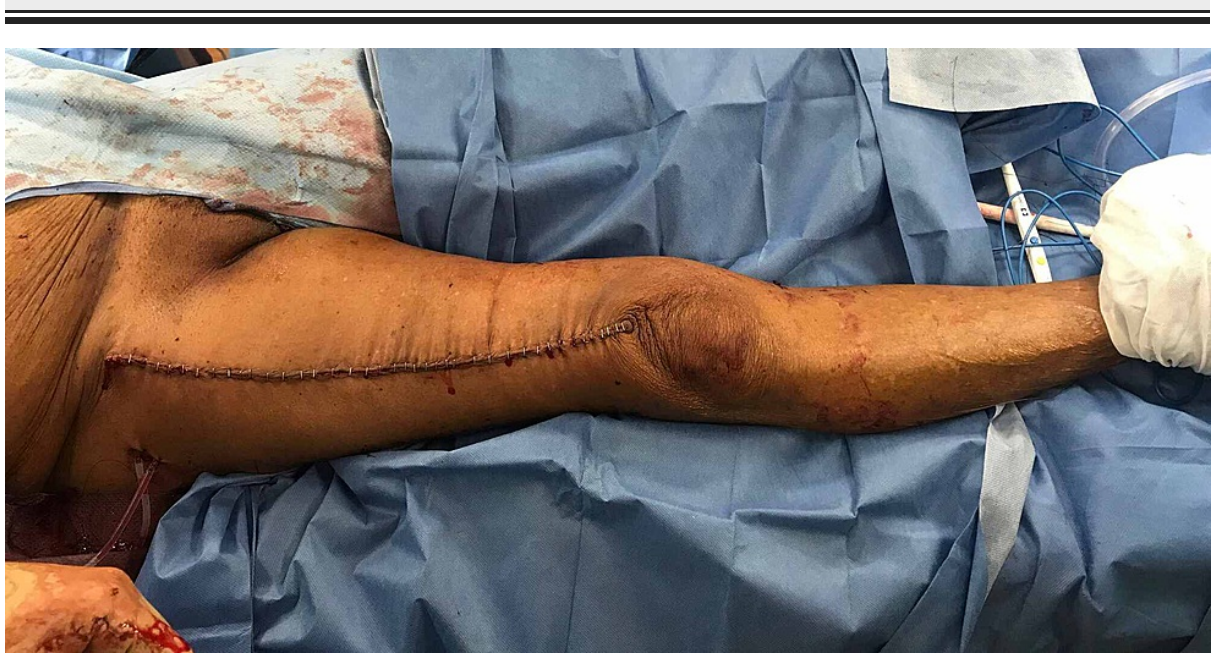

FIGURE 8: Primary closure of the anterior right thigh.

Pathologic examination revealed the mass to measure $30 \times 20 \times 15 \mathrm{~cm}$ and weigh 4,607 $\mathrm{g}$ (Figures 9-11) . Sectioning revealed a marbled yellow cut surface with interspersed cystic and firm/fibrotic white areas (Figure 12). Fat necrosis was seen along the periphery. No lymph nodes were identified. The central non- 


\section{Cureus}

lipogenic component of the tumor measured $20 \times 15 \times 14 \mathrm{~cm}$ with a mitotic rate of five mitoses per $10 \mathrm{HPF}$ and was classified as Fédération Nationale des Centres de Lutte Contre le Cancer histologic grade 2 . The tumor was classified as American Joint Committee on Cancer pathologic stage III (T4NOMOG2). Scattered necrosis was present in $10 \%$ of the tumor. All margins were negative for tumor invasion, with the closest margin being $<1 \mathrm{~mm}$, posteriorly. MDM2 gene amplification was positive by FISH. These findings indicated a DDL of the right anterior thigh.

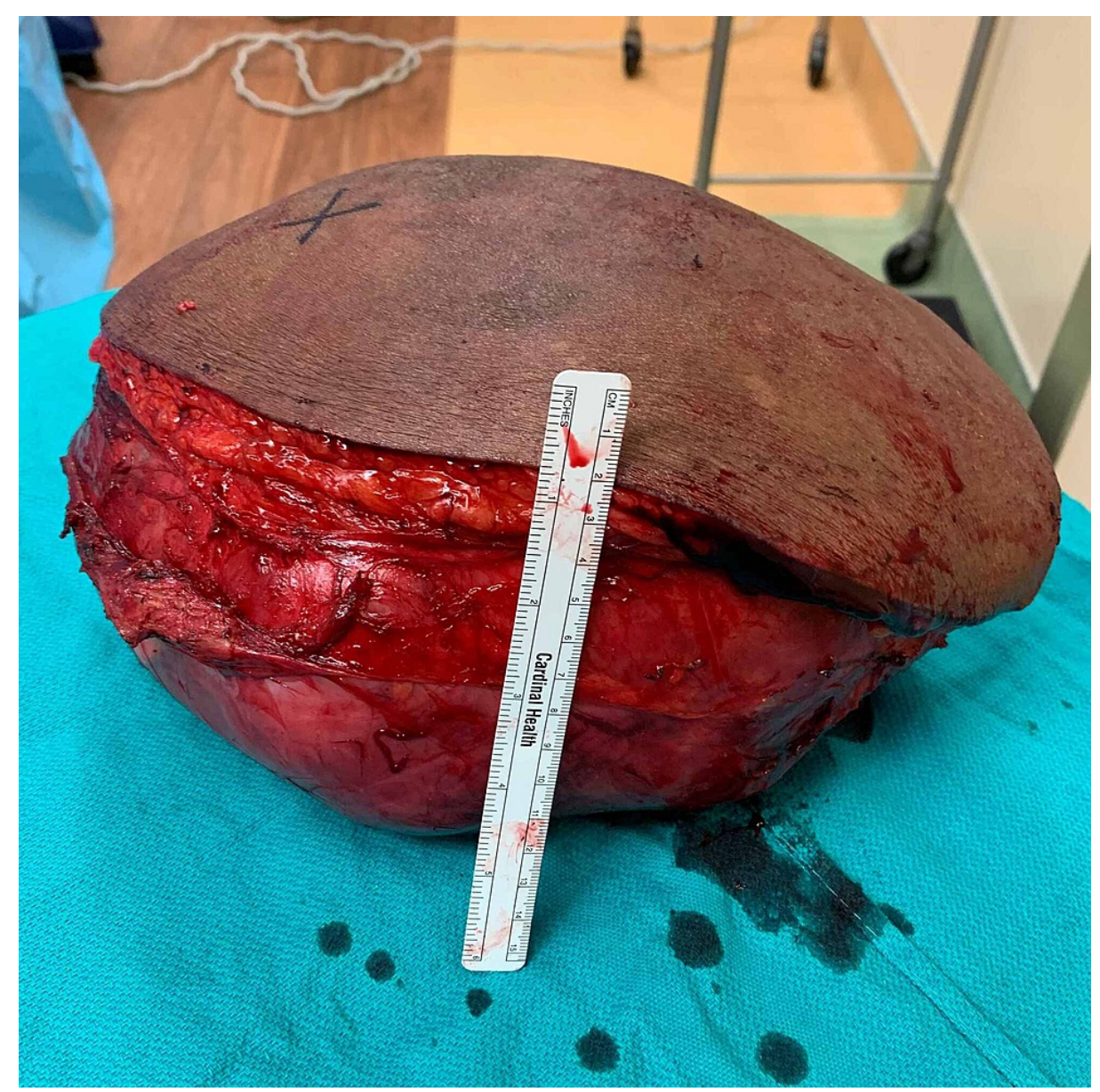

FIGURE 9: Macroscopic tumor appearance: anterior-medial view. 


\section{Cureus}

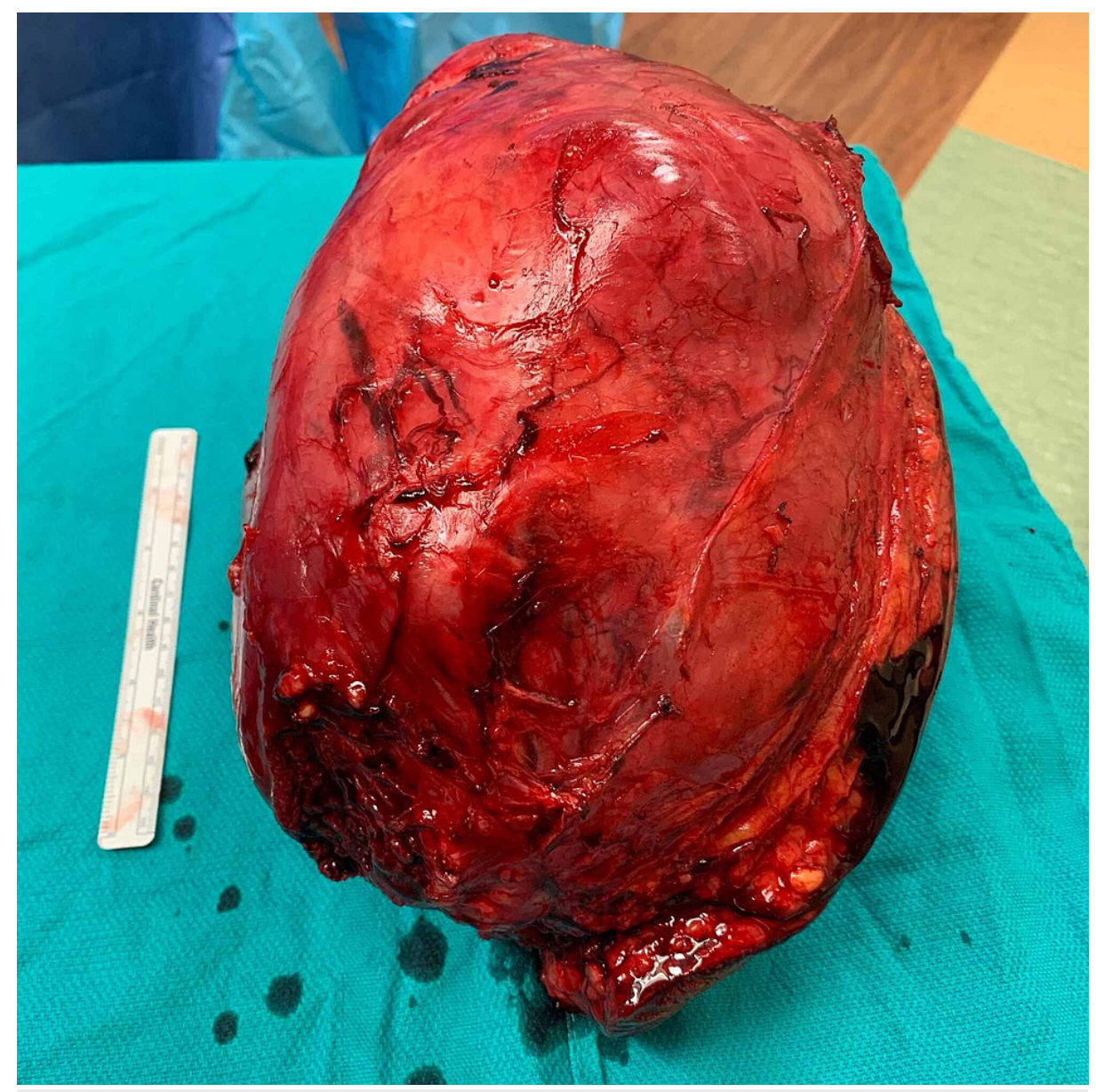

FIGURE 10: Macroscopic tumor appearance: medial view. 


\section{Cureus}

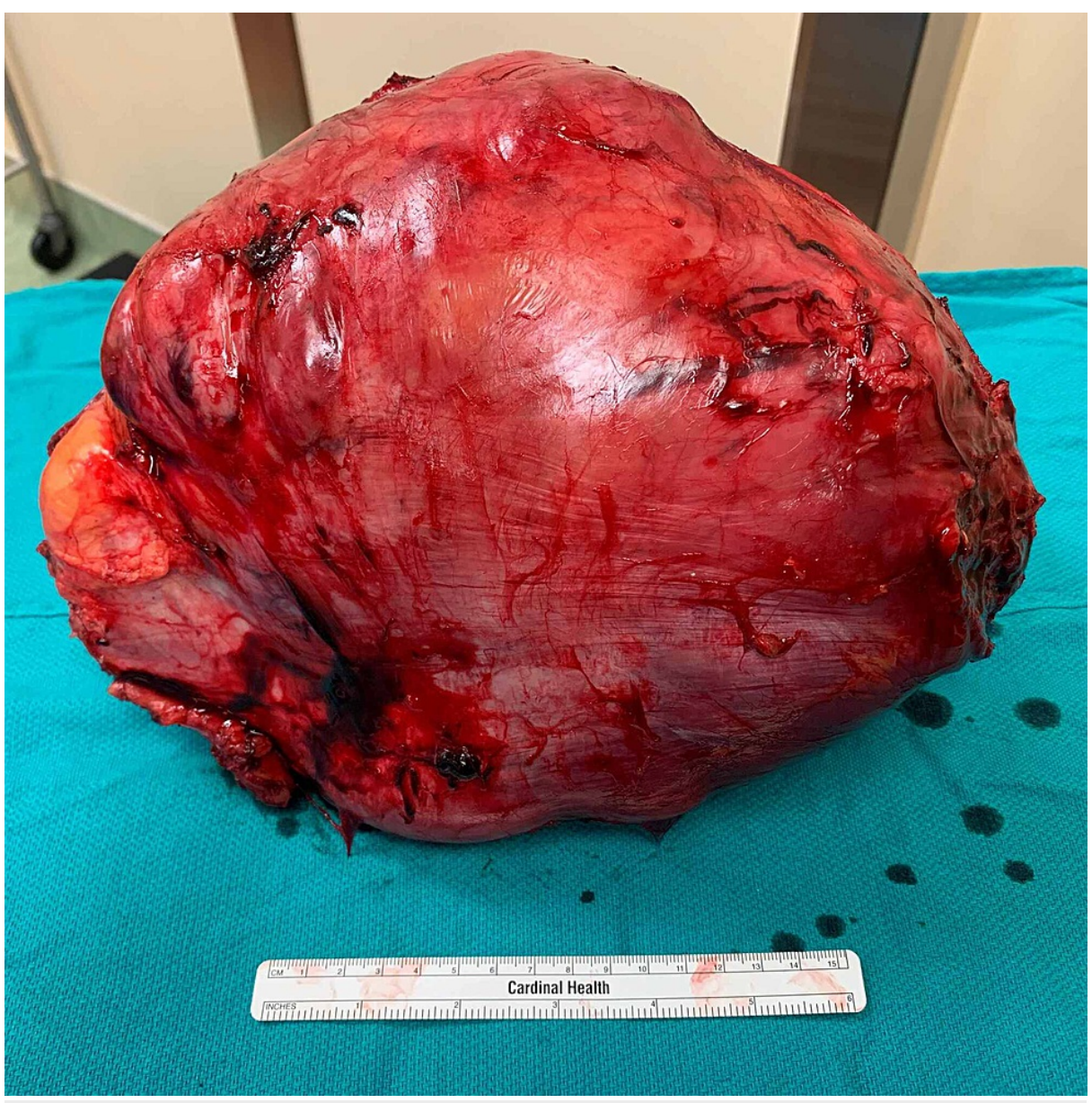

FIGURE 11: Macroscopic tumor appearance: posterior view.

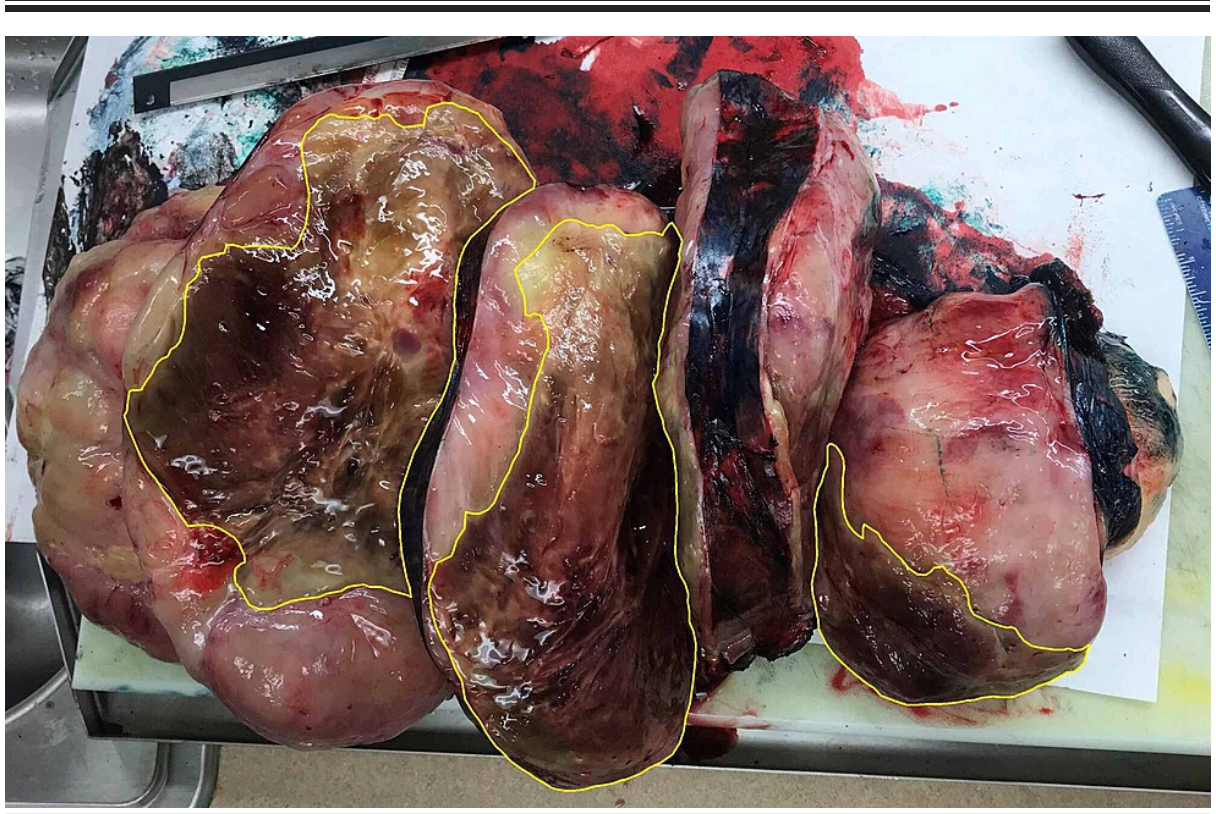

FIGURE 12: Longitudinal cross-sections of the tumor. Sectioning revealed a marbled fatty surface with areas of necrosis. There is a central lobulated mass measuring $20 \times 15 \times 14 \mathrm{~cm}$. Within the central mass are firm and fibrotic nodular areas intermixed with foci of cystic degeneration and necrosis (yellow outlines). 


\section{Cureus}

\section{Outcome}

The patient's post-operative recovery was uneventful. On post-operative day 2, her range of motion in the right hip improved to 15-120 degrees (from 30-105 degrees) as well as in the right knee to 30-120 degrees (from 75-105 degrees). Muscle strength in the right lower extremity improved with respect to hip flexion, hip extension, knee flexion, and knee extension. By post-operative day 4, the patient demonstrated continued improvement in right knee extension and was able to ambulate with a rolling walker. The patient was discharged on post-operative day 5 and closely followed throughout completion of her adjuvant radiotherapy and physical therapy. The patient received intensity-modulated radiation therapy to the anterior right thigh with bolus to the surgical scar. Over eight weeks, 61.2 Gray in 34 fractions was administered to both the superior and inferior portions of the anterior right thigh. Based on the surgical margin status, the patient received an additional 11.2 Gray of radiation to the 50 Gray recommended for patients without pre-operative radiation therapy [8]. This was well-tolerated except for hyperpigmentation and mild desquamation of her skin. Despite the narrow margins, the primary resection combined with radiotherapy was deemed sufficient treatment for the patient's liposarcoma. At one month post-operatively, the patient was able to ambulate with the assistance of a cane.

\section{Discussion}

Liposarcomas are soft tissue tumors that are generally classified into four subtypes: WDL, DDL, myxoid liposarcoma, and pleomorphic liposarcoma [4]. Both WDL and DDL commonly arise in the extremities and retroperitoneum but rarely in the mediastinum, para-testicular region, or spinal cord [2,3]. Liposarcomas located in the retroperitoneum are often diagnosed at a later stage compared to those in extremities and other superficial soft tissues, which may explain to some extent the higher rates of local recurrence and metastasis of retroperitoneal tumors $[2,3]$.

DDL is a non-lipogenic sarcoma that is thought to arise as a progression of WDL [2]. The risk of dedifferentiation to DDL is directly related to the location and duration of tumor growth [2]. About $10 \%$ of DDL arise as a recurrence of WDL, while nearly $90 \%$ of DDL occur within a primary WDL lesion, most commonly in the retroperitoneum [2,5]. Unlike WDL, DDL has a propensity for metastasis, and a greater incidence of local recurrence after resection, resulting in decreased overall and disease-free survival (Table 1) [9]. Mortality from DDL is most often due to uncontrolled local recurrent disease than metastasis [2]. However, metastasis is an indicator of poor prognosis with a five-year survival rate of $<18 \%$ in patients with stage IV tumors [6]. The most common sites of metastasis in descending order include the lungs, subcutaneous soft tissues, lymph nodes, and liver [10]. The median time to metastasis is approximately eight months in patients initially presenting with localized DDL [6]. The five-year survival rate for stage I and stage II DDL is approximately $65 \%$, which reduces to $49 \%$ for stage III and < $18 \%$ for stage IV (Table 2) [6].

\begin{tabular}{|c|c|c|}
\hline Liposarcoma subtype & WDL & DDL \\
\hline Local recurrence rate (\%) & 23 & 38 \\
\hline Metastatic rate (\%) & 3 & 14 \\
\hline 5-year overall survival (\%) & 92 & 54 \\
\hline
\end{tabular}

TABLE 1: Local recurrence/metastatic rate and five-year overall survival for extremity liposarcoma by subtype.

WDL, well-differentiated liposarcoma; DDL, dedifferentiated liposarcoma. Data adapted from Vos et al. [9] 


\section{Cureus}

Survival rates of DDL

5 years (\%)

AJCC stage

Stage I

63

Stage II

67

Stage III

49

Stage IV

18

FNCLCC grade

Grade 1

69

Grade 2

Grade 3

Primary site

Head/Neck

Extremities

Pelvis

Thorax/Trunk

Retroperitoneum/Abdomen

TABLE 2: Survival rates of DDL by AJCC stage, FNCLCC grade, and primary site.

DDL, dedifferentiated liposarcoma; AJCC, American Joint Committee on Cancer; FNCLCC, Fédération Nationale des Centres de Lutte Contre le Cancer. Adapted from Gootee et al. [6]

Primary surgical resection with negative margins with or without subsequent neoadjuvant and adjuvant radiotherapy is recommended for soft tissue sarcomas of the extremities, superficial trunk, and head and neck areas that are resectable with acceptable functional outcomes [8]. Resection should achieve negative margins $>1.0 \mathrm{~cm}$ and/or intact fascial planes [8,11]. Marginal resection is associated with increased rates of local recurrence, metastasis, and decreased five-year survival (Table 3) [12]. Radiotherapy is not recommended for treatment of WDL as it is considered radio-insensitive [11,13]. DDL of the extremities $>5$ $\mathrm{cm}$ and those with close or positive margins are generally treated with adjuvant radiotherapy, especially if treatment of local recurrence would likely compromise limb function [11,13]. All patients who did not receive pre-operative radiation therapy should receive 50 Gray of radiotherapy with a boost dose of 10-16 Gray for negative margins, 16-18 Gray for microscopically positive margins, and 20-26 Gray for gross residual disease [8]. However, re-resection is preferred for patients with positive surgical margins [8]. For DDL of the retroperitoneum, radiotherapy is limited in use as it has not been shown to improve overall survival $[11,13]$. WDL and DDL are generally resistant to chemotherapy, but there may be a role in treatment for other subtypes of liposarcoma [2,3]. Management of DDL must always include frequent follow-up to assess for local recurrence and lung metastasis with MRI of the primary tumor site every six months and chest imaging every 6-12 months [8,11]. 


\section{Cureus}

10.1097/PAS.0000000000000366

8. Mehren MV, Randall RL, Benjamin RS, et al.: Soft tissue sarcoma, version 2.2018, NCCN Clinical Practice Guidelines in Oncology. J Natl Compr Canc Netw. 2018, 16:536-563. 10.6004/jnccn.2018.0025

9. Tirumani SH, Tirumani H, Jagannathan JP, Shinagare AB, Hornick JL, Ramaiya NH, Wagner AJ: Metastasis in dedifferentiated liposarcoma: predictors and outcome in 148 patients. Eur J Surg Oncol. 2015, 41:899904. 10.1016/j.ejso.2015.01.012

10. Vos M, Koseła-Paterczyk H, Rutkowski P, et al.: Differences in recurrence and survival of extremity liposarcoma subtypes. Eur J Surg Oncol. 2018, 44:1391-1397. 10.1016/j.ejso.2018.03.028

11. Muratori F, Frenos F, Bettini L, et al.: Liposarcoma: clinico-pathological analysis, prognostic factors and survival in a series of 307 patients treated at a single institution. J Orthop Sci. 2018, 23:1038-1044. 10.1016/j.jos.2018.06.008

12. Crago AM, Dickson MA: Liposarcoma: multimodality management and future targeted therapies . Surg Oncol Clin N Am. 2016, 25:761-773. 10.1016/j.soc.2016.05.007

13. Crago A, Singer S: Clinical and molecular approaches to well differentiated and dedifferentiated liposarcoma. Curr Opin Oncol. 2011, 23:373-378. 10.1097/CCO.0b013e32834796e6

14. Stevenson M, Musters A, Geertzen J, van Leeuwen B, Hoekstra H, Been L: Amputations for extremity soft tissue sarcoma in an era of limb salvage treatment: local control and survival. J Surg Oncol. 2017, 117:434442. 10.1002/jso.24881

15. Alamanda V, Crosby S, Archer K, Song Y, Schwartz H, Holt G: Amputation for extremity soft tissue sarcoma does not increase overall survival: a retrospective cohort study. Eur J Surg Oncol. 2012, 38:1178-1183. 10.1016/j.ejso.2012.08.024

16. Smith H, Thomas J, Smith M, Hayes A, Strauss D: Major amputations for extremity soft-tissue sarcoma . Ann Surg Oncol. 2017, 25:387-393. 10.1245/s10434-017-5895-2

17. Misra A, Mistry N, Grimer R, Peart F: The management of soft tissue sarcoma . J Plast Reconstr Aesthet Surg. 2009, 62:161-174. 10.1016/j.bjps.2008.08.018 